\title{
Estructura como envolvente en la iglesia nuestra señora del carmen - cúcuta
}

\section{Structure as an envelope in the nuestra señora del carmen church - cúcuta.}

\author{
Yannette Díaz Umaña ${ }^{1}$ \\ Julio Alfredo Delgado Rojas ${ }^{2}$ \\ Mawency Vergel Ortega ${ }^{3}$ \\ Universidad Francisco de Paula Santander.
}

\section{RESUMEN}

Este artículo de investigación en arquitectura sagrada tiene como objetivo analizar la geometrización de las formas laminares de hormigón de la iglesia de Nuestra Señora del Carmen en San José de Cúcuta. Este proyecto de estudio descriptivo, analiza las variables del diseño arquitectónico para la Arquitectura Moderna, con especial énfasis en las condiciones particulares que hacen de la cubierta en hormigón una estructura liviana, 1 Magister en Gestión Urbana, Arquitecta, docente y directora del Departamento de Arquitectura, diseño y Urbanismo. Filiación: Universidad Francisco de Paula Santander. Correo electrónico: yannettedu@ufps.edu.co Orcid: https://orcid.org/0000-0003-4582-1593

2 Magister en Educación Matemática, Arquitecto, Docente. Filiación: Universidad Francisco de Paula Santander. Correo electrónico: julioalfredo@ufps.edu.co Orcid: https://orcid.org/0000-0001-6944-832X

3 Doctora en Educación. Postdoctora en Imaginarios y representaciones sociales. Docente y Directora del Departamento de Matemáticas y Estadística. Filiación: Universidad Francisco de Paula Santander. Correo electrónico: mawency@ufps.edu.co. Orcid: https://orcid.org/0000-00018285-2968 y con cualidades excepcionales al trabajo en voladizo. Las losas de concreto conforman una plegadura prismática semiradial, de modo que las fuerzas internas se distribuyen a lo largo de sus bordes, dando resistencia a todo el sistema, un ejemplo del rigor geométrico al trabajo de la estructura.

\section{PALABRAS CLAVES}

Arquitectura moderna sagrada, geometría, plegadura prismática, triangulo.

\section{SUMMARY}

This research article in sacred architecture aims to analyze the geometrization of the concrete laminar forms of the church of Nuestra Señora del Carmen in San José de Cúcuta. This descriptive study project analyzes the variables of architectural design for Modern Architecture, with special emphasis on the particular conditions 
that make the concrete roof a light structure, with exceptional qualities for cantilever work. The concrete slabs form a semi-radial prismatic folding, so that the internal forces are distributed along its edges, giving resistance to the whole system, an example of geometric rigor to the work of the structure.

Translated with www.DeepL.com/Translator (free version)Key words: Modern sacred architecture, geometry, prismatic folding, triangle.

\section{INTRODUCCIÓN}

Las formas laminares en hormigón, son también conocidas como la técnica estructural de plegaduras, que conforman sistemas tridimensionales con la unión de sus aristas en ángulos. La rigidez del conjunto se da por la forma de los componentes y su unión, de modo que las delgadas láminas de hormigón armado se caracterizan por sus perfiles planos articulados con bordes pronunciados. A diferencia de las láminas curvas cuya característica está dada por su arqueamiento simple o de doble curvatura. Históricamente se les reconoce por su alta capacidad de rigidez especialmente en voladizos.

Con referencia a sus orígenes Saldarriaga (2015) comenta:

"Las láminas plegadas fueron utilizadas por vez primera en Alemania, en 1924, siendo empleadas por Ehlers en la construcción de grandes depósitos de carbón. Ehlers publicó su primer trabajo sobre láminas plegadas en el año 1930. Sin embargo, este tipo de estructuras alcanzó su mayor importancia y desarrollo a partir de 1947, cuando los ingenieros americanos se interesaron en el tema y aplicaron posteriormente los ordenadores electrónicos a los métodos de análisis, tanto a los ya existentes, como a los que posteriormente fueron apareciendo." (Pág. 33)
Posteriormente su auge se da, entre los años 50 y los años 70 del siglo XX. Al respecto, comenta García, (2007) que entre las estructuras plegadas más reconocidas está la sala de conferencias de la UNESCO en París construida por Nervi, Breuer y Zehrfuss y el hangar de Alleghany Airlines en el aeropuerto de Boston que tiene una luz de 75,86m. Por su parte Gonzales, Sanmartín, y Martinez (s.f.), se refieren al Seminario Mayor en Medellín como un caso de orden nacional.

Con referencia a sus características, se dice que estas plegaduras delgadas presentan menores requerimientos con los trabajos del encofrado, como lo afirma Angerer en García (2.013) con referencia a las de superficie en hormigón curva. No obstante, para ambos tipos de estructura, Coca (2.014) manifiesta que el control y calidad en sus técnicas de elaboración, es exhaustiva, además de requerir formaletas y andamiajes especiales con una cantidad precisa de unidades, por lo que la mano de obra debe ser capaz en número y habilidad para construcción de estas formas laminares.

En correspondencia con las características de estos sistemas de plegaduras Díaz (2019) las define como superficies continuas, que por medio de su rigidez lograda con los pliegues se consigue mayor resistencia, de allí que el aumento de pliegues, cambia sus propiedades estructurales. En este sentido se advierte que el plegado hace que la distribución de masas optimice notablemente su comportamiento, aumentando el momento de inercia de la sección, que en orientación longitudinal puede asimilarse a una viga como lo argumenta Delaloye (2.008). En lo que se refiere a las clases de superficies plegadas, Coca (2.014) y Delaloye (2.008) armonizan en una clasificación sencilla que abarca varias tipologías, concentrándolas en cuatro géneros: las prismáticas, antiprismáticas, piramidales y trapezoidales. 
En el caso de la iglesia de Nuestra Señora del Carmen, objeto de estudio de esta investigación, cuya cubierta en estructura de lámina plegada pertenece al tipo prismática, se puede interpretar el trabajo como viga (pliegue) y el funcionamiento transversal como losa (superficie lisa). Para Delaloye (2.008) las losas con las cargas propias de su material y las sobrecargas como la lluvia, granizo y personal de mantenimiento. Estas se apoyan sobre las aristas o pliegues que funcionan como vigas, dicho de otra forma, el cordón superior e inferior que actúan como vigas, soportan las armaduras (losas) principales, mientras que a lo largo de esta superficie se absorben sus esfuerzos cortantes (Cassinello 1.996). De ahí es importante que esta articulación no se pierda para que estas reacciones de la losa deban ser absorbidas correctamente por las vigas. En este caso Coca (2.014) recomienda el uso de elementos de borde y tímpanos rigidizadores para mantener la forma. (imagen 1)

Imagen 1. Vista aérea de la estructura laminar en hormigón de la iglesia de Nuestra Señora del Carmen.

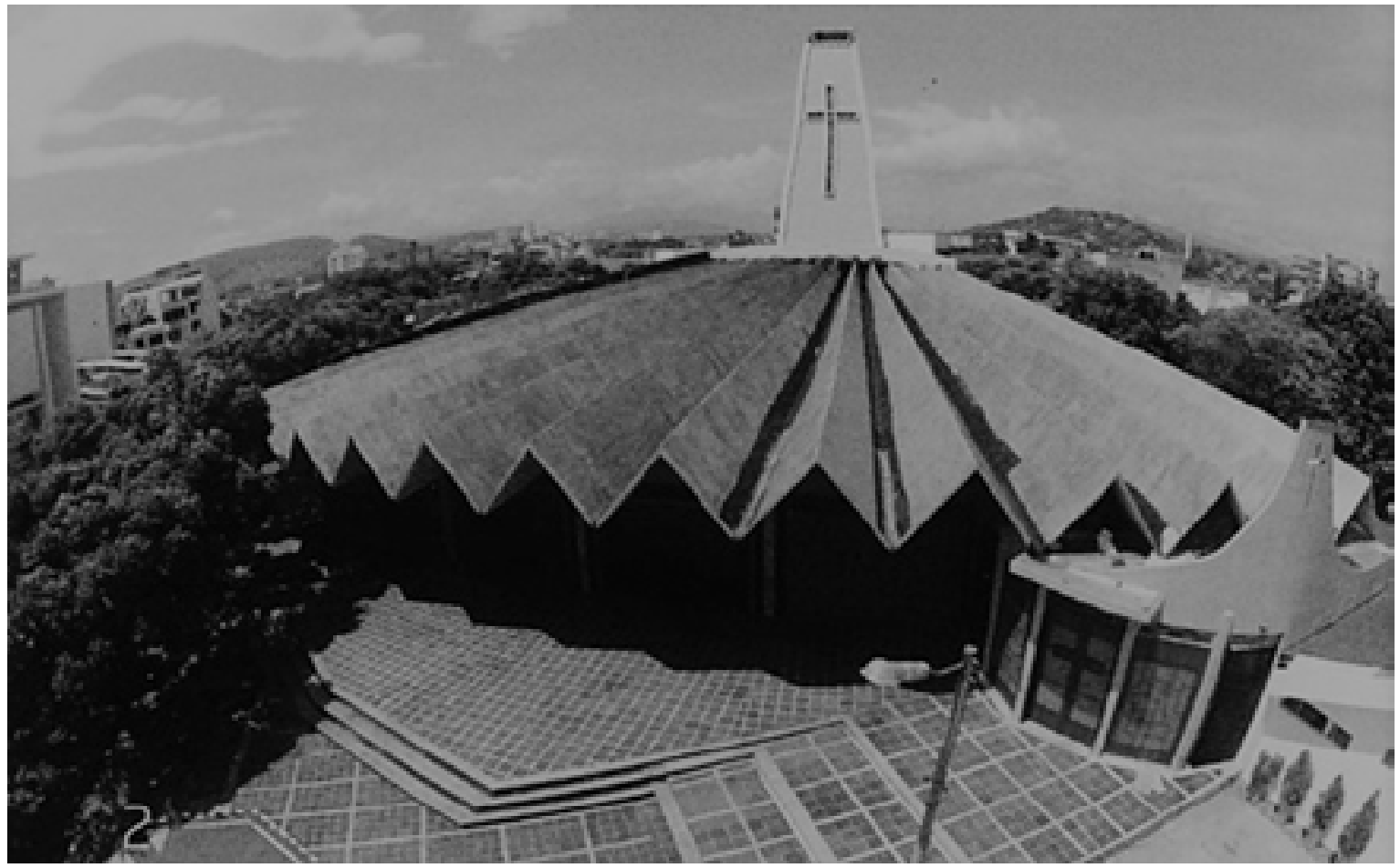

Fuente. Revista Cromos (2.012).

\section{DISCUSIÓN}

Dada estailustración, la iglesia de Nuestra Señora del Carmen se desarrolla bajo una cubierta con sistema de superficie activa en lámina plana articulada por arista o plegada, con una luz libre de 35m., cuyas líneas de intersección forman las correspondientes limatesas y limahoyas, formando 14 secciones triangulares similares en su vista frontal. Se trata, por tanto, de un tipo estructural que permite salvar grandes luces pese a su escaso espesor. Su forma, en plegadura, le presta rigor transversal, como lo explica Baldanta (2015), quien observa, que la arista que se forma en el pliegue, alcanza una rigidez enorme a compresión, siendo los planos que la forman los que impiden que esta pandee. De esta manera el comportamiento longitudinal 
de estas, son como las de una viga apoyada en los extremos, a su vez, transversalmente, funciona como una viga continúa apoyada en los pliegues, con menor rigidez. (Imagen 2)

Imagen 2. Esquema de la forma de plegaduras prismáticas lineales, radiales y semiradial con relación a la distribución de las cargas.
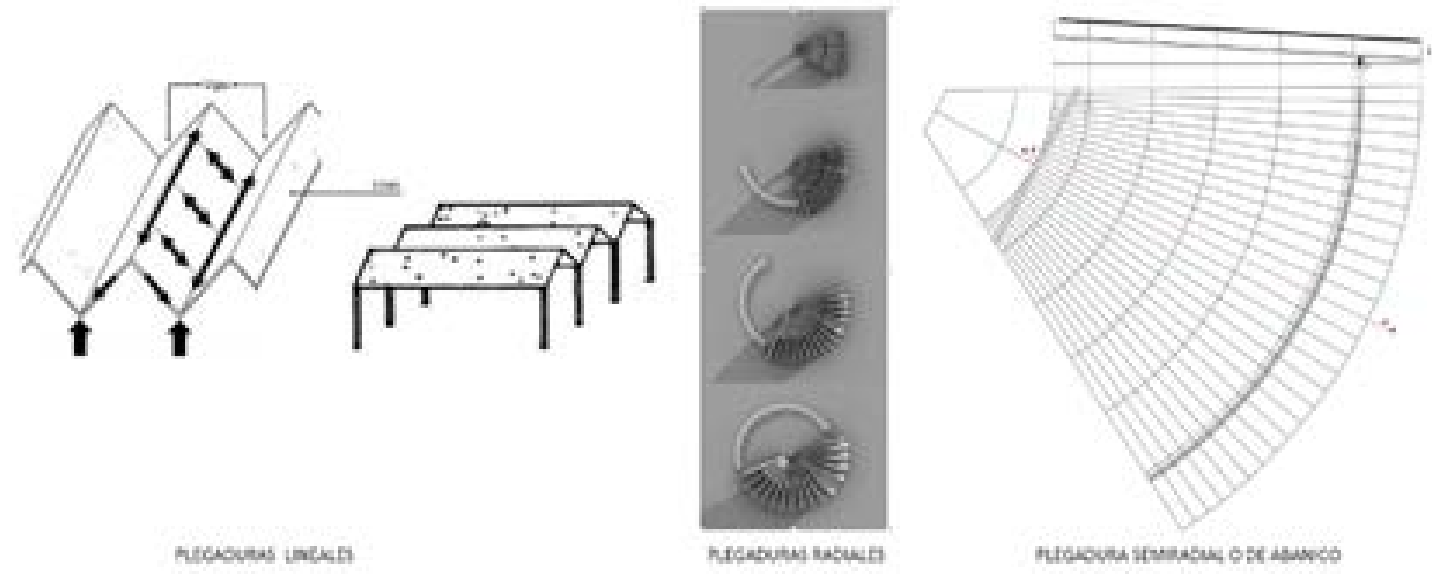

Fuente: Elaboración propia.

Esta plegadura en hormigón, constituye una estética muy particular, en vista del conjunto de formas estructurales que se conciben paralelamente unas a otras en forma de abanico. Mujica (2015) en este aspecto se presenta dos subcategorías, las lineales cuando el módulo de plegado se da en una sola dirección, y las plegaduras radiales cuando los módulos se desarrollan en torno a un centro, ambas clases se comportan estructuralmente de forma similar, ya que su perfil básico en triangulo es el mismo para ambos casos.

Por consiguiente, la iglesia de Nuestra Señora del Carmen, responde a la codificación en sistema laminar semiradial, ya que su origen se da en puntos muy próximos que consolidan esa direccionalidad en forma de abanico. De este modo, la envolvente se apoya sobre sobre el altar debajo de la torre y sobre el pórtico de la fachada. Su longitud sobrepasa la fachada principal por lo que ofrece protección de los rayos del sol al atrio, dejando ver el voladizo, parte del atractivo estético y funcional de este tipo de envolventes.

No hay que desconocer, las propiedades constructivas y plásticas del hormigón, que le adjudican a este sistema de superficie activa, como también se le conoce, un buen funcionamiento estructural. Diaz (2019), menciona a Cassinello (1.996) al rememorar el carácter "formaceo" y "adecuorrecistente" en las estructuras plegables gracias al uso del hormigón convertido en estructura a la vista, de forma óptima y con capacidad suficiente para resistir las cargas con esfuerzos internos reducidos, alcanzando espesores mínimos. De allí, que este autor, se refiera a la liberación del peso para dar mayor libertad formal y espacial de estas estructuras plegables. No obstante, también se advierte el reto para calcularlas y los prejuicios estéticos ante nuevas formas, limitando la aplicación, que no supero las dos décadas a nivel mundial (García 2007). 
Observando la composición arquitectónica de la iglesia estudiada, se presentan tres componentes ordenadores, el perimetral conformado por los muros, y pilares, la cubierta en forma de plegadura, y la torre, participando de las labores estructurales y estableciendo racionalmente la distribución de espacios tanto interiores como exteriores. Esta proyección le da protagonismo sin lugar a dudas, a la forma de la cubierta, encargada de liberar el espacio interno. Se muestra de forma clara su ligereza y capacidad de soportar la carga con un mínimo de material al considerar la sección de la membrana en hormigón. (imagen 3)

Imagen 3. Corte de la iglesia de Nuestra Señora del Carmen.

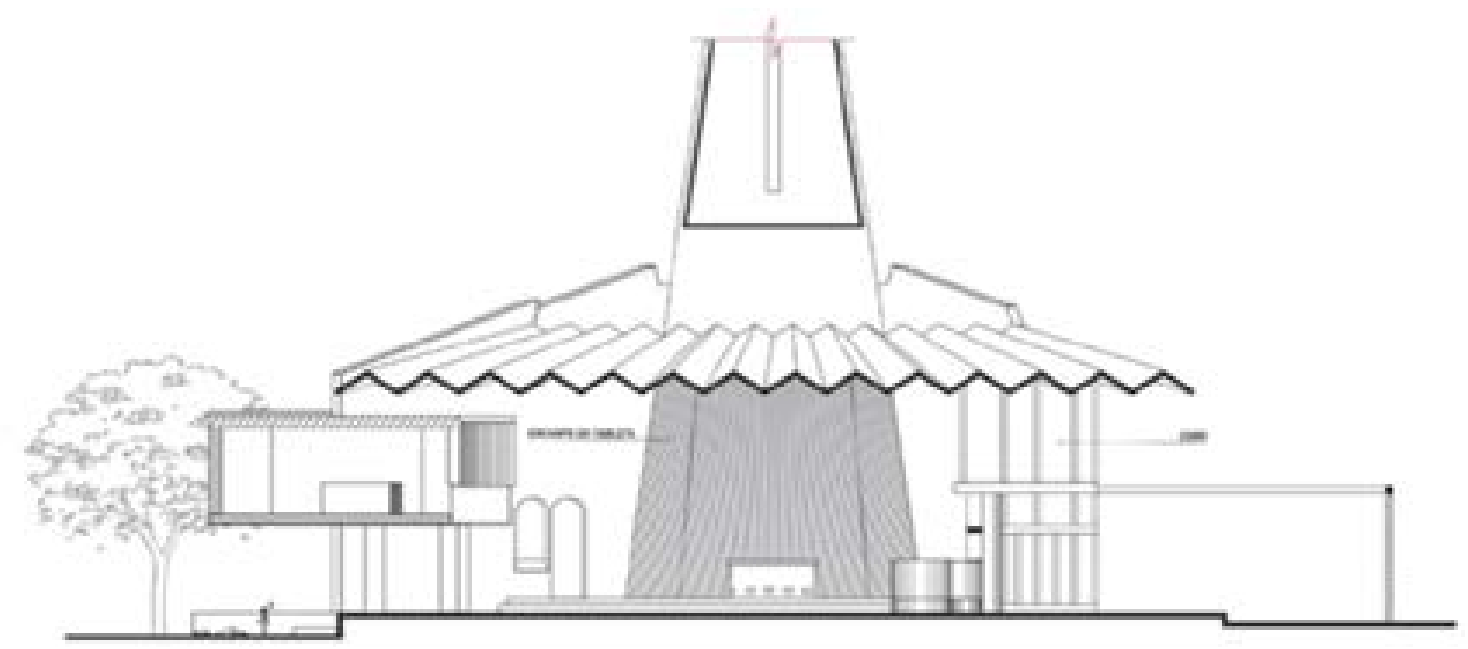

CORTE TRANSVERSAL A - A___ALTAR \& CORO

Fuente: estudiantes María Camila Acuña, Jose Arbey, Jesús Álvarez.

En particular la geometría fundamentada en la forma simple de la plegadura, crea el sistema de superficie activa que posibilita la estructura de la iglesia de Nuestra Señora del Carmen, donde el rigor geométrico, conceptúa la correlación entre elementos y por tanto las fuerzas de cohesión. De este modo las losas de concreto conforman una geometría regular, de modo que las fuerzas internas se distribuyen a lo largo de sus bordes, dando resistencia a todo el elemento. Esta sincronía de agrupaciones geométricas posibilita el funcionamiento de la estructura. Si se acepta que el perfil en forma de triángulos que se alcanzan a ver en su interior, son parte de esta geometría, se podrá entender la rigidez de todo el sistema.
Explicando esta condición desde el punto de vista estructural, García (2007) enseña que existe una condición establecida que concede distribuir las cargas actuantes dosificándolas por toda la superficie mediante esfuerzos de pequeña magnitud. El uso de pliegues habilita un cubrimiento horizontal, y una eficiencia en la resistencia vertical a las fuerzas gravitacionales. Estas placas consienten cubrir luces mayores que las losas, que funcionan como estructuras de masa activa. Se puede decir que es un sistema genuino de forma al trabajo de la estructura, donde los pliegues mismos crean una superficie activa, muy delgada en hormigón, cuyos nervios recubiertos que son la armadura, no requieren más de tres centímetros de protección. 
En cuanto a la forma de sustentación de la losa se realiza desde el pliegue inferior de la lámina, que se apoya en dos partes, generando la sensación de un abanico que se posa sobre los muros de ladrillo. No obstante, el sistema de vigas de soporte descansa sobre dos líneas de columnas ubicadas en el frente y contrafrente del templo, de tal manera que la fachada principal y la base de la torre consolidan el rol estratégico de la estructura portante en el diseño y se visualiza en la forma como se contrae el abanico hacia el contrafuerte y se amplía en el frente. Las secciones generadas en perfil de esta estructura, son los 14 triángulos isósceles de $1.8 \mathrm{~m}$. de dimensión en dos de sus lados, y de $2 \mathrm{~m}$. la base. Esta figura geométrica, conseguida con el mismo pliegue, permite apreciar la morfología de la cubierta, al dejar correr la luz de manera mística como herramienta de la arquitectura sagrada (Vergel, Delgado, \& Díaz (2020).

Profundizado en la forma principal que compone la plegadura, correspondiente al triángulo Isósceles, reconocido como: "Es el que tiene dos lados congruentes" (Guerrero, 2006, p.31), se identifican, los ángulos opuestos a estos lados también como congruentes o de la misma medida. De allí, la altura que cae sobre la base es al mismo tiempo mediana y bisectriz del ángulo opuesto a la base. A su vez la altura correspondiente a la base divide al triángulo en dos triángulos rectángulos iguales, tal como lo afirma Delgado (2008). Así se comprende que “ [...] en un triángulo isósceles la altura sobre la base es a la vez mediana del triángulo y bisectriz del ángulo opuesto" (Guerrero (2006,p.108). Por su parte, la bisectriz, que es la línea que biseca un ángulo del triángulo y va al lado opuesto, son determinadas por (Guerrero, 2006, p.90) como "Las bisectrices de los ángulos congruentes de un triángulo isósceles son congruentes" en otras palabras, como los ángulos tienen la misma medida al igual que los lados, cualquiera de las dos líneas trazadas bajo las mismas condiciones debe medir igual. (Imagen4)

Imagen 4. Geometría básica del triángulo del perfil de plegaduras prismáticas semiradial en la iglesia de Nuestra Señora del Carmen.
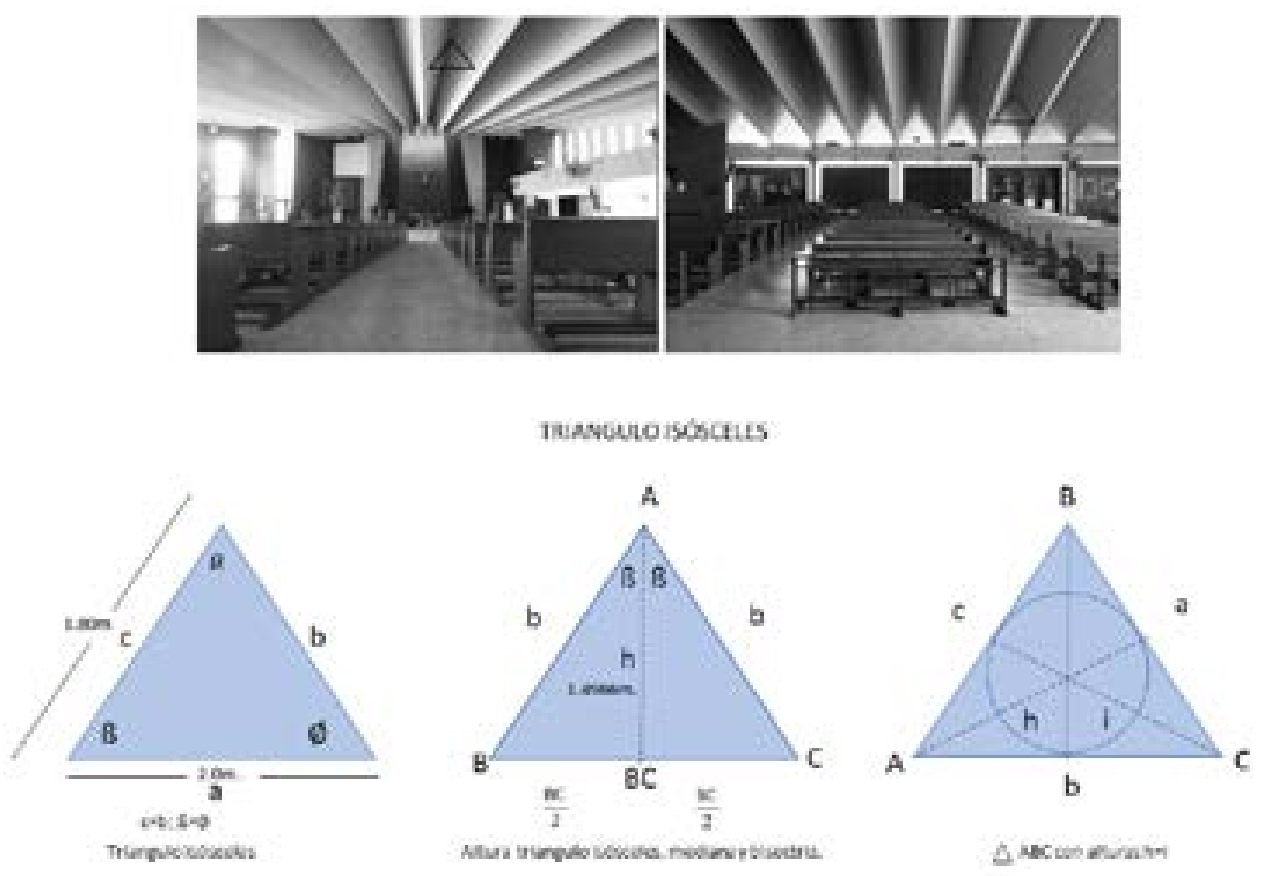

Fuente: elaboración Propia. 
Todavía falta mencionar, que el contrafuerte se dispone con un arco de descarga, que permite que todo el peso de la pared se traslade a ambos lados del vano, denominadas las jambas. Estéticamente estas jambas acompañan simétricamente la torre vistas desde el interior de la iglesia. Es así como este elemento tan característico de las iglesias preconciliares, la torre (Díaz, Delgado \& Vergel 2021) de $21.5 \mathrm{~m}$ de alto, confiere orden al esquema general de la iglesia, dando jerarquía, pues su sistema de pliegues paralelos parece originarse desde este punto. Paradójicamente, esta estructura no posee campanario, acogiéndose al Concilio Vaticano II, que influyo en la desaparición de los campaneros hacia la década de los sesenta, atendiendo los sistemas mecanizados de sonidos (Delgado, Díaz, y Vergel 2019). En este sentido se podría afirmar que la torre abandona su función primaria de campanario, para acoger un rol constructivo, estructural, morfológico y simbólico que acompaña la estructura laminar de hormigón prismática de forma semiradial.

Esta composición denota como la arquitectura moderna hace uso de la geometría, para imprimir al espacio sagrado un ambiente místico, alcanzado con la cubierta en plegadura de tipo prismático semiradial. Algo semejante ocurre con el uso de las cúpulas en la arquitectura sagrada medieval, cuyo significado cosmológico, en el sentido artístico y constructivo, al denotar la zona presbiteral y la ingravidez representando el mismo cielo (Díaz, Delgado \& Vergel 2021). Después de todo esta superficie en hormigón también revela unas propiedades plásticas y constructivas, haciendo notoria la jerarquía ascendente hacia el presbítero, mostrada en la direccionalidad de los pliegues. La rigidez por su parte, reconoce su propiedad casi ingrávida, al dotar al espacio de una gran luz sin apoyos a través de los haces de iluminación provenientes de su forma básica el triángulo, igualmente con sentido simbólico.

\section{CONCLUSIÓN}

En ultimas la iglesia de Nuestra señora del Carmen, posee una cubierta con sistema de superficie activa, en plegadura de tipo prismático, semiradial que proyecta una simbiosis entre la obra arquitectónica y su estructura. Se puede decir que es un sistema genuino de forma al trabajo de la estructura, donde los pliegues mismos crean una superficie activa, muy delgada en hormigón, con nervios recubiertos que son la armadura. En otras palabras, sin el rigor geométrico, no habría correlación entre elementos y por tanto no habría fuerzas de cohesión.

Así mismo, se debe destacar el papel del hormigón, en este tipo de soluciones constructivas, ya que es un estupendo mediador entre las iniciativas plásticas apoyadas en la geometría y las ambiciones de tipo estructural que fueron consideradas para esta obra. Con esto la capacidad plástica, constructiva y estructural, así como también, la idoneidad para resolver la concepción de arquitectura sagrada, al unificar aspectos como materialidad, sencillez y continuidad espacial, otorgan a esta iglesia una cualidad excepcional en el referente nacional e internacional de arquitectura con un sistema estructural de superficie activa en Hormigón. En definitiva, se logró, una estética bastante llamativa, solo comparable con obras emblemáticas de la arquitectura moderna internacional, solventando necesidades locales referentes al uso, la tipología, y la estructura.

\section{REFERENCIAS BIBLIOGRÁFICAS}

Baldanta, C. (2015). Análisis de la estructura de la Universidad Laboral de Tarragona (E.Torroja). http://hdl.handle.net/10251/55265.

Cassinello, F. (1.996). Construcción en hormigonería. Segunda edición. Editorial Rueda. Madrid. 
Coca, S. (2.014). Planteamiento de uniones dinámicas para elementos tubulares con base en la morfología de sistemas de superficie activa (plegaduras). Tesis Maestría. Universidad Nacional de Colombia. Facultad de artes,escuela de arquitectura y urbanismo. Bogotá. Visto en: http://www.bdigital.unal.edu.co/56674/7/ SindyM.CocaNeusa.2014.pdf

Delaloye, H. (2.008). Estructuras - Nivel 4 taller: vertical III. Guía de Estudio N³: Láminas Plegadas. Universidad Nacional de la Plata Facultad de Arquitectura y Urbanismo. Visto en http://www.tallerdnc.com.ar/index.php/niveles/ plan-v/nivel-iv/descargas/category/29-guiasestudio?download=103\%3Age- 3

Delgado, Alfredo; Díaz, Yannette y Vergel, Mawency (2019). El Paisaje Arquitectónico y sonoro del Campanario de la Catedral de San José de Cúcuta. Revista Logos Ciencia \& Tecnología, vol. 11, no. 1, (2019):52-60. Consulta en diciembre 10 2019: file:///C:/Users/ ufps/Downloads/801-Texto\%20del\%20art\%C3 \%ADculo-4700-1-10-20190126.pdf

Delgado, R. (2008). Matemática familiar. Fundamentos de 6 a 11 y pensamiento lógico. Manizales, Colombia: Editores S.A.

Díaz, Yannette (2019). La arquitectura sagrada en San José de Cúcuta (II Parte): Caso de estudio, Iglesia de Nuestra Señora del Carmelitas. Cúcuta. Trabajo investigativo presentado para el escalafón docente. Repositorio Universidad Francisco de Paula Santander.

Díaz Umaña, Y., Delgado Rojas, J. A., \& Vergel Ortega, M. (2021). Representaciones socioespaciales, en la memoria histórica de la catedral y su entorno urbano en San José de Cúcuta. Revista Boletín Redipe, 10(4), 376-387. https://doi.org/10.36260/rbr.v10i4.1277

Díaz-Umaña Y, Vergel-Ortega M, Delgado-Rojas JA. (2020) Modelo geométrico y arquitectónico de la cúpula mayor en San José de Cúcuta. bol. redipe [Internet]. 1 de marzo de 2020 [citado 6 de mayo de 2021];9(3):160-6. Disponible en: https://revista.redipe.org/index.php/1/article/ view/941

García, R. (2.013). Dos décadas de estructuras plegadas de hormigón. Inicio y ocaso de un movimiento. Informes de la Construcción Vol. $65,529,27-39$, enero-marzo visto en: http:// informesdelaconstruccion.revistas.csic.es/ index.php/informesdelaconstruccion/article/ viewFile/2610/2922

García, Rafael (2007). Láminas plegadas de hormigón armado. Realizaciones en España. Actas del Quinto Congreso Nacional de Historia de la Construcción, Burgos, 7-9 junio 2007, eds. M. Arenillas, C. Segura, F. Bueno, S. Huerta, Madrid: I. Juan de Herrera, SEdHC, CICCP, CEHOPU, 2007. Visto en: https:// www.researchgate.net/publication/322642330 Laminas_plegadas de hormigon_armado Realizaciones_en_Espana

Gonzales, Jose; Samartín, Avelio y Martinez, Jesús (s.f.). Evolución de los métodos de cálculo de láminas plegadas. Una aportación al análisis de estructuras no prismáticas" visto en : https://core.ac.uk/download/pdf/148670707.pdf

Guerrero, B. (2006). Geometría. Desarrollo axiomático. Bogotá, Colombia. Ecoe Ediciones

Montano, G. (2013). Geometría elemental. Honduras. Tegucigalpa. Guaymuras.

Mujica, Alfredo. (2015). Estructuras Ligeras - Plegaduras. Apuntes de arquitectura digital. blogspot visto en : http:// apuntesdearquitecturadigital. blogspot. com/2015/06/estructuras-ligeras-plegaduras. $\mathrm{html}$

Saldarriaga, Melisa (2015). Viabilidad técnica y económica de la construcción de cascarones de hormigón. Trabajo de grado para optar al título de ingeniera civil. Escuela de Ingeniería de 
Antioquia ingeniería civil. Envigado Colombia. Visto en : https://repository.eia.edu.co/bitstream/ handle/11190/2042/RamirezMelisa_2015 Viabilidad TecnicaEconomica. pdf?sequence $=1 \&$ isAllowed $=y$

Mujica, Alfredo. (2015). Estructuras Ligeras - Plegaduras. Apuntes de arquitectura digital. blogspot visto en : http:// apuntesdearquitecturadigital.blogspot. com/2015/06/estructuras-ligeras-plegaduras. $\underline{\mathrm{html}}$

Vergel Ortega, M.., Delgado Rojas, J. A.., \& Díaz Umaña, Y. (2020). Estudio iconográfico y geométrico del vitral de San José en la catedral de Cúcuta. Revista Boletín Redipe, 9(6), 119133. https://doi.org/10.36260/rbr.v9i6.1006 